Case report

\title{
Fatal infection with Taenia martis metacestodes in a ring-tailed lemur (Lemur catta) living in an Italian zoological garden
}

\author{
Claudio De Liberato ${ }^{a}, *$, Federica Berrilli ${ }^{b}$, Roberta Meoli ${ }^{a}$, Klaus G. Friedrich ${ }^{c}$, Pilar Di Cerbo ${ }^{c}$, \\ Cristiano Cocumelli ${ }^{a}$, Claudia Eleni ${ }^{\mathrm{a}}$ \\ a Istituto Zooprofilattico Sperimentale delle Regioni Lazio e Toscana, Via Appia Nuova 1411, 00178 Rome, Italy \\ b Dipartimento di Medicina Sperimentale e Chirurgia, Università di Roma Tor Vergata, Via Montpellier 1, 00133 Rome, Italy \\ ${ }^{c}$ Fondazione Bioparco, Viale del Giardino Zoologico, 100197 Rome, Italy
}

\section{A R T I C L E I N F O}

\section{Article history:}

Received 31 March 2014

Received in revised form 20 May 2014

Accepted 30 May 2014

Available online 10 June 2014

\section{Keywords:}

Taenia martis

Lemur catta

Larval cestode

Zoological garden

Italy

\begin{abstract}
A B S T R A C T
A case of fatal infection caused by larval forms of Taenia martis in a ring-tailed lemur (Lemur catta) living in the Rome zoological garden is described. The animal, living in a semi-natural pen with other 15 conspecific individuals and being fed with fresh fruit and vegetables, yoghurt and eggs, was transported to the Istituto Zooprofilattico of Rome for post-mortem examination. The anamnesis included, ten days before the death, apathy, lack of appetite, abdominal distension and diarrhoea. A severe exudative fibrinous-purulent peritonitis with numerous adhesions between the abdominal wall and the bowel loops was detected. After intestine removal, two free and viable, $4 \mathrm{~cm}$ long, whitish, leaf-like parasitic forms were pinpointed. Macroscopic examination of the two parasites allowed their identification as larval stages of cestodes, identified via molecular analysis as T. martis metacestodes. This report represents the first record of $T$. martis infection in the host species and in a zoological garden and for the pathological relevance of the infection.
\end{abstract}

(c) 2014 Elsevier Ireland Ltd. All rights reserved.

\section{Introduction}

Taenia martis (Zeder, 1803) (Cestoda, Cyclophyllidea) is a tapeworm parasite of Mustelidae, with a widespread distribution in the northern hemisphere. Its intermediate hosts are mainly arvicoline and murine rodents. In Europe, adults of this species have been recorded, usually with low prevalence, in Martes martes (pine marten), Martes foina (stone marten) and Mustela putorius (European polecat) [1-3], while Clethrionomys glareolus (bank vole), Microtus agrestis (field vole), Apodemus sylvaticus (wood mouse) and Ondatra zibethicus (muskrat) act as intermediate hosts [3-5]. Higher prevalence has been recorded in M. martes in Spain (5.88\%) [3] and in O. zibetichus in Belgium (22.2\%) [5]. In Italy, at present, $T$. martis has been reported in M. foina from North-Western (prevalence 33.3\%) and Southern Italy [1,6] and in C. glareolus from Calabria Region (Southern Italy) [7]. A case of fatal infection with larval forms of $T$. martis in a ring-tailed lemur (Lemur catta) from the zoological garden (Bioparco) of Rome is described here. The Bioparco is one of the oldest zoological gardens in Europe, founded in 1911. It is located in the city centre on an area of 18 ha, housing more than 1000 animals belonging to almost 200 species. The reported case was considered to be interesting as: 1) it represents the first record of $T$. martis larval forms in $L$. catta; 2 ) it is the first record of this parasite in a zoological garden, raising the question

\footnotetext{
* Corresponding author. Tel.: + 39 0679099336; fax: + 390679099331.

E-mail address: claudio.deliberato@izslt.it (C. De Liberato).
}

about the possible transmission routes; and 3) the pathological relevance of the infection, considered responsible for the animal death, maybe due to the unusual host/parasite association.

The lemur, an 18-year-old male, used to live in a semi-natural pen with other 15 conspecific individuals and was transported in June 2013 to the Istituto Zooprofilattico Sperimentale delle Regioni Lazio e Toscana of Rome for post-mortem examination. The ring-tailed lemur colony was introduced at the Bioparco in 2007, arriving from the zoo of Ostrava (Czech Republic). Animals are fed with fresh fruit and vegetables, yoghurt and eggs. The anamnesis included, ten days before the death, apathy, lack of appetite, abdominal distension and diarrhoea. Metronidazole (12.5 mg/kg, P.O. q12 h) and spiramycin (75,000 UI/kg, P.O. q12 h) for 7 days and streptomagma ( $1 \mathrm{ml}$, P.O. q12 h) for 10 days were administered. After an initial improvement, the animal showed a decrease in appetite and a tendency to isolate itself from the group until death.

At the post-mortem examination, a severe exudative fibrinouspurulent peritonitis was observed (Fig. 1a), with numerous fibrous adhesions between the abdominal wall and the bowel loops and the haemorrhagic suffusions. Intestine showed a marked wall thickening and congestion of a $5 \mathrm{~cm}$ segment of the colon, with inward large necrotic-haemorrhagic areas and purulent exudates on the mucosa (Fig. 1b). The cecum and liver presented constipation and degeneration, respectively. No macroscopic lesions were detected in other organs. Two viable parasitic forms approximately $4 \mathrm{~cm}$ long, whitish and leaflike were observed free in the peritoneal cavity (Fig. 1c). Based on their morphology, the two parasites were recognised as cestode larval 


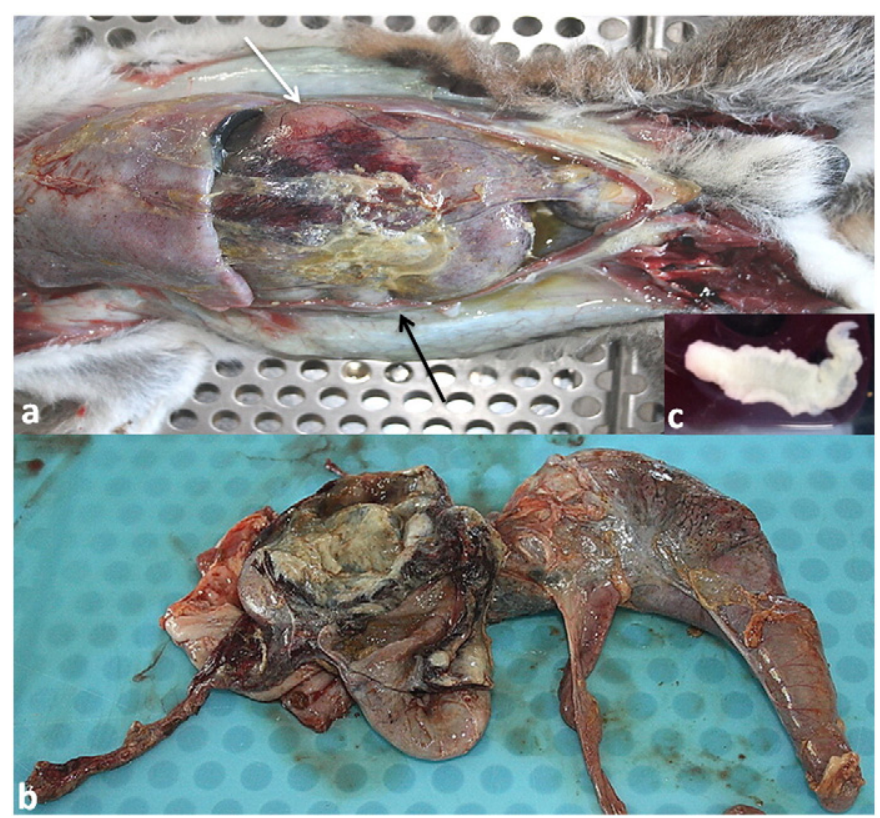

Fig. 1. a) Peritoneal cavity: exudative fibrinous-purulent peritonitis with haemorrhagic suffusions (white arrow) and fibrous adhesions (black arrow); b) Left: colon with thickened wall and necrotic-haemorrhagic mucosa. Right: constipated cecum; c) Taenia martis larva.

forms and transferred for molecular identification at the University of Rome "Tor Vergata".

Samples of the lung, heart, liver, spleen, kidney, colon and brain were routinely processed for histopathology. Microscopic examination of colon showed extensive infiltrates of neutrophils, lymphocytes, plasma cells and eosinophils in the mucosa, with numerous necrotic-haemorrhagic foci associated with haemosiderosis. The muscle layers revealed large areas of fibrous proliferation and disseminated haemorrhages. Multifocally, inflammatory infiltrates extended from the mucosa to the peritoneal serosa, where many degenerate neutrophils and few eosinophils were detected. In absence of any evidence of other causes, such as a foreign body or a neoplastic lesion, it could be speculated that these findings were due to the passage of the oncospheres through the intestinal epithelium of an unsuitable host. No significant microscopic lesions were observed in the other examined organs, besides hepatocyte degeneration, a light liver cholestasis and small perivascular haemorrhages in the heart and brain.
Bacteriological examination of the intestine, liver and peritoneal exudates did not reveal any bacterial agent, probably due to the administration of spiramycin.

Genomic DNA from one specimen was extracted using the QIAamp Mini Kit (Qiagen) according to the manufacturer's protocol. A portion of the mitochondrial cytochrome oxidase I gene (cox1) was amplified by PCR using the primers JB3 and JB4.5 [8]. Bands of the predicted sizes were excised and DNA was purified and directly sequenced on both strands by the Bio-Fab Research (Rome, Italy). To assign the isolate to the specie level, the obtained sequence was compared with available sequences in GenBank database by using the Standard Nucleotide BLAST search. To test the significance of the result, a phylogenetic analysis was also performed by comparison of the obtained sequence with those of reference strains using the Neighbour joining method ( $\mathrm{NJ}$ ) (bootstrap test 1000 replicates) by MEGA5 [9]. The sequence obtained in this study was deposited in the GenBank database and is available under the accession number: KJ459910. Cox1 sequence analyses unambiguously identified the larva as belonging to $T$. martis species, the sequence showing the highest identity (99\%) with those of all $T$. martis isolates available in GenBank, while significantly lower identity with other tapeworm species was observed, e.g. Taenia twitchelli (94\%), Taenia hydatigena (86\%-89\%), Taenia multiceps (86\%). A well-defined cluster supported by significant bootstrap values (98\%) and corresponding to T. martis isolates including sequence obtained from $L$. catta, was evidenced in the NJ tree (Fig. 2), thus confirming species assignment.

To the best of our knowledge, this is the first report of T. martis metacestodes in the host species and in any host living in a zoo. The ability of tapeworm larval forms to infect a highly diverse range of host species is well documented in literature. Interestingly, a case of infection by Echinococcus multilocularis metacestodes in a ring-tailed lemur living in a zoo was recently reported in France [10]. Regarding T. martis, larval forms of this species were recorded in wild cats [11] and in 2013 a human infection was reported in Germany [12]. In the present case, the two larvae were alive and moving at the moment of their recovery from the $L$. catta peritoneal cavity. Maybe because of the unusual host/parasite association, clinical and pathological changes observed in the lemur were very severe and the peritonitis could have provoked a state of severe toxaemia, such as to compromise the vital functions and to determine the lemur death. Regarding the transmission route in the Bioparco, two main possibilities have to be taken into consideration: a) the lemur was already infected when the colony was introduced in the Rome zoological garden in 2007; b) T. martis eggs arrived in the pen with the fresh vegetables daily fed to the lemurs. Considering the pathological changes observed, it is unlikely that the

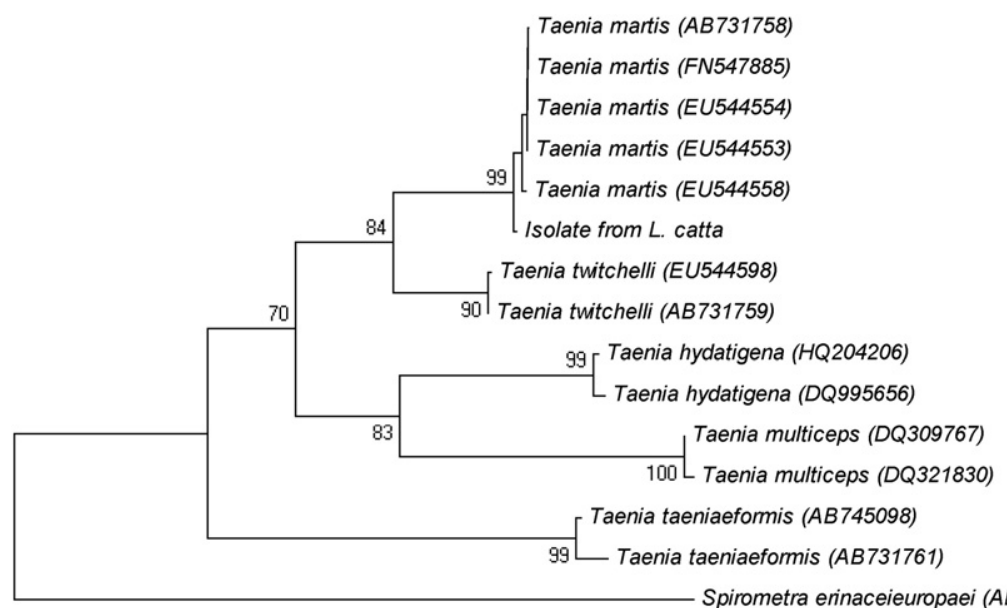

Spirometra eninaceieuropaei (AB015754)

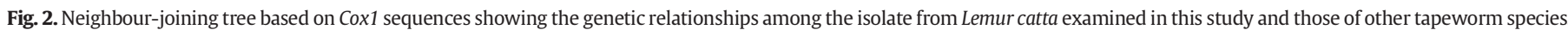
GenBank accession numbers of reference strains are in parentheses. 
animal lived for 8 years with the two metacestodes in the peritoneal cavity. On the other hand, T. martis was frequently recorded in M. foina in Italy. This mustelid is quite common in the country, and contamination of vegetables with infected faeces must be retained a plausible hypothesis. A similar explanation was also suggested for the human case recorded in Germany [12]. Assuming that the parasite entered the zoological garden as eggs contaminating fresh vegetables, its zoonosic potential should be taken into consideration. T. martis metacestodes can infect humans [12] and the possibility of zoonotic infections via contaminated fresh vegetables cannot be ruled out, even in an urban context where the cycle of such a parasite is not expected.

\section{References}

[1] Millán J, Ferroglio E. Helminth parasites in stone martens (Martes foina) from Italy. Z Jagdwiss 2001;47:229-31.

[2] Shimalov VV, Shimalov VT. Helminth fauna of the European polecat (Mustela putorius Linnaeus, 1758) in Belorussian Polesie. Parasitol Res 2002;88:259-60.
[3] Segovia JM, Torres J, Miquel J, Sospedra E, Guerrero R, Feliu C. Analysis of the helminth communities of the pine marten, Martes martes, in Spain: mainland and insular data. Acta Parasitol 2007;52:156-64.

[4] Fuentes MV, Sainz-Elipe S, Galàn-Puchades MT. Ecological study of the wood mouse helminth community in a burned Mediterranean ecosystem in regeneration five years after a wildfire. Acta Parasitol 2007;52:403-13.

[5] Mathy A, Hanosset R, Adant S, Losson B. The carriage of larval Echinococcus multilocularis and other cestodes by the musk rat (Ondatra zibetichus) along the Ourthe river and its tributaries (Belgium). J Wildl Dis 2009;45:279-87.

[6] Ribas A, Milazzo C, Foronda P, Casanova JC. New data on helminths of stone marten, Martes foina (Carnivora, Mustelide) in Italy. Helminthologia 2004;41:59-61.

[7] Milazzo C, Casanova JC, Aloise G, Ribas A, Cagnin M. Helminths of the bank vole Clethrionomys glareolus (Rodentia, Arvicolinae) in Southern Italy. Ital J Zool 2009;70:333-7.

[8] Bowles J, Blair D, McManus DP. Genetic variants within the genus Echinococcus identified by mitochondrial DNA sequencing. Mol Biochem Parasitol 1992;54:165-73.

[9] Tamura K, Dudley J, Nei M, Kumar S. MEGA4: molecular evolutionary genetics analysis (MEGA) software version 4.0. Mol Biol Evol 2007;24:1596-9.

[10] Umhang G, Lahoreau J, Nicolier A, Boué F. Echinococcus multilocularis infection of a ring-tailed lemur (Lemur catta) and a nutria (Myocastor coypus) in a French zoo. Parasitol Int 2013;62:561-3.

[11] Schuster R, Heidecke D, Schierhorn K. Contributions to the parasite fauna of local hosts. 10. On the endoparasitic fauna of Felis silvestris. Appl Parasitol 1993;34:113-20.

[12] Eberwein P, Haeupler A, Kuepper F, Wagner D, Kern WV, Muntau B, Racz P, Agostini H, Poppert S. Human infection with marten tapeworm. Emerg Infect Dis 2013;19:1152-4. 